\title{
Karotis-OP im Alter riskanter
}

Fragestellung: Welchen Einfluss haben in Deutschland Alter und Geschlecht auf die Sterblichkeit und die Schlaganfallrate im Krankenhaus bei Patienten, die sich einer Karotis-OP unterziehen?

Hintergrund: Der therapeutische Nutzen der Operation und des Stentings einer hochgradigen symptomatischen Karotisstenose zur Schlaganfallprävention ist unumstritten. Weiterhin umstritten ist aber der therapeutische Nutzen der Operation von asymptomatischen Stenosen, insbesondere bei einer Lumeneinengung unter $70 \%$ ohne Progredienz. Die ursprünglichen Studien zum Nutzen einer Operation von asymptomatischen Karotisstenosen liegen lange zurück. In dieser Zeit war eine optimale Therapie der wichtigen vaskulären Risikofaktoren beispielsweise mit Angiotensin-Rezeptorblockern bei Hypertonie oder Statinen bei Hypercholesterinämie nicht verfügbar. In der Folgezeit wurden dann nur noch Studien durchgeführt, bei denen die Karotis-OP mit dem Stenting verglichen wurde.

Patienten und Methodik: Es handelt sich um eine Analyse des Qualitätsregisters der deutschen Gefäßchirurgie. Erfasst wurden 142.074 Karotis-OP, die zwischen 2009 und 2014 durchgeführt wurden. Der primäre Endpunkt der Analyse waren Schlaganfall und Tod bis zur Krankenhausentlassung.

Schmid S, Tsantilas P, Knappich $C$ et al. Risk of inhospital stroke or death is associated with age but not sex in patients treated with carotid endarterectomy for asymptomatic or symptomatic stenosis in routine practice: Secondary data analysis of the nationwide German statutory quality assurance database from 2009 to 2014. J Am Heart Assoc 2017; 6: pii: e004764
Ergebnisse: Das Register erfasste 142.074 Karotis-OP, davon 88.200 aufgrund asymptomatischer Stenosen. 68 \% der Patienten waren Männer, das mittlere Alter betrug 71 Jahre. Der primäre Endpunkt trat bei 1,8\% der Frauen und 1,9\% der Männer ein. Sowohl das Operationsrisiko als auch die Mortalität waren mit dem Alter, nicht aber mit dem Geschlecht assoziiert.

Schlussfolgerungen: Zunehmendes Alter war mit einem erhöhten Risiko von Schlaganfall und Tod im Krankenhaus assoziiert, nicht jedoch das Geschlecht der Patienten.

\section{- Kommentar von Hans-Christoph Diener, Essen}

\section{Noch mehr Zurückhaltung geboten}

Der Referent hat die vorliegende Publikation nicht wegen ihrer Analyse der Datenbank herausgesucht, sondern wegen der erschreckend hohen Zahl von 88.200 Personen, die zwischen 2009 und 2014 an einer asymptomatischen Karotisstenose operiert wurden. Die Indikation für die Operation einer asymptomatischen Karotisstenose ist durch die S3-Leitlinie der Deutschen Gesellschaft für Gefäßchirurgie und der Deutschen Gesellschaft für Neurologie abgesichert. Die Numbers needed to treat ist allerdings sehr hoch. Völlig unberücksichtigt bleibt bisher, ob eine aggressive konservative Behandlung nicht einer operativen oder interventionellen Therapie überlegen ist. Dies konnte zumindest für symptomatische intrakranielle Stenosen gezeigt werden. Die deutsche SPACEII-Studie musste leider wegen schlechter Rekrutierung vorzeitig beendet werden. Die Studie zeigt aber, dass eine aggressive konservative Therapie mindestens genauso wirksam ist wie die Karotis-OP oder das Stenting. Diese Ergebnisse würden darauf hindeuten, dass man mit Eingriffen bei asymptomatischen Karotisstenosen in Zukunft noch zurückhaltender sein sollte als bisher. 